\title{
GENOTIPIFICACIÓN Y SUBTIPIFICACIÓN MOLECULAR DE CEPAS DE Clostridium perfringens AISLADAS EN ALPACAS MUERTAS POR ENTEROTOXEMIA
}

\author{
Molecular GenOtyPing and SUbTYPING OF CLOSTRIDIUM PERFRINGENS IsOLATED \\ FROM Fatal ENTEROTOXEMIA IN Alpacas
}

\author{
David Pérez ${ }^{1}$, Lenin Maturrano ${ }^{2,4}$, Raúl Rosadio ${ }^{2,3}$
}

\section{RESUMEN}

\begin{abstract}
A pesar de que la enterotoxemia causada por el Clostridium perfringens ocasiona elevadas tasas de mortalidad neonatal en alpacas, muy poco se conoce acerca de las toxinas que participan en la patogénesis de la enfermedad. El presente estudio reporta resultados de análisis moleculares de 47 cepas de C. perfringens aislados de casos mortales de enterotoxemia en alpacas, basados en la amplificación de los genes $c p a, c p b$, etx y iap codificantes de las toxinas $\alpha, \beta$, g, e $\overline{3}$ (genotipificación), así como la detección de genes secundarios codificantes de la enterotoxina (сpe) y toxina $\beta 2$ (subtipificación). El ADN bacteriano de las 47 cepas fue sometido a prueba de PCR múltiple evidenciando que 46/47 (97.9\%) de los aislamientos correspondieron al genotipo A y de estos, 13/46 (28.3\%) contenían adicionalmente el gen $\beta 2$ (subtipo $c p e^{-\mathrm{vo}} c p b 2^{+\mathrm{vo}}$ ), mientras que las restantes 33 muestras $(71.7 \%)$ fueron negativas para ambos genes secundarios (subtipo $\left.c p e^{-\mathrm{vo}} c p b 2^{-\mathrm{vo}}\right)$. Solo una de las 47 (2.1\%) muestras correspondió al genotipo C y positivo al gen CPE (subtipo $c p e^{+\mathrm{vo}} \mathrm{cpb2} 2^{-\mathrm{vo}}$ ). Estos resultados sugieren la posible participación de la toxina alfa y beta 2 en los casos de enterotoxemia de las alpacas, pero excluyen a la enterotoxina.
\end{abstract}

Palabras clave: Clostridium perfringens, genotipificación, enterotoxemia, alpacas

\section{Abstract}

Although enterotoxemia produced by Clostridium perfringens causes elevated neonatal mortality in the alpaca, scarce information exists on the virulence factors (toxins) which play an important role in the pathogenesis of the disease. The objective of this study was to genotype and subtype $C$. perfringens isolates from enterotoxemia induced mortalities based on the presence of genes $c p a, c p b$, etx and iap which encode the toxins $\alpha, \beta$, g, and $\overline{3}$, and the genes cpe and cpb2 which encode the enterotoxin (cpe) and $\beta 2$ -

\footnotetext{
1 Servicio Nacional de Sanidad Agraria - SENASA, Perú

${ }^{2}$ Unidad de Biología y Genética Molecular, Facultad de Medicina Veterinaria, Universidad Nacional Mayor de San Marcos, Lima

${ }^{3}$ CONOPA - Instituto de Investigación y Desarrollo de Camélidos Sudamericanos, Perú

${ }^{4}$ E-mail: lenin.maturrano@gmail.com
} 
toxins, respectively. A total of 47 C. perfringens isolates were obtained from the small intestine of neonatal alpaca mortalities which presented clinical signs, as well as gross and histological injuries typical of enterotoxemia. The results showed that 46/47 (97.9\%) of the isolates were genotype A, 13 of these 46 (28.3\%) also had cpb2 (genotype A, subtype cpe $^{-}$cp $\left.^{+}\right)$, while the remaining $33(71.3 \%)$ were negative for both cpe and $c p b 2$ genes (genotype A, subtype cpe cpb2-) and only $1 / 47(2.1 \%)$ had the cpa, cpb у сре genes (genotype $\mathrm{C}$, subtype $c p e^{+} c p b 2^{-}$). The results indicate that both the alpha and beta2 toxins likely play an important role in enterotoxemia aetiology, but excluded participation of the enterotoxin.

Key words: Clostridium perfringens, genotyping, enterotoxemia, alpacas

\section{INTRODUCCIÓN}

La enterotoxemia en el Perú es la principal enfermedad infecciosa que afecta a las alpacas (Vicugna pacos) neonatas. Moro (1966) la describió por primera vez, mencionando al Clostridium perfringens, principalmente del tipo A, y en algunos casos al tipo $\mathrm{C}$, como el agente causal. La enfermedad tiene un comportamiento epizoótico, alcanzando tasas de mortalidad neonatal de 50\% en varios centros de crianza alpaquera del sur del Perú (Carbajal, 1974; Ramírez et al., 1985; Ameghino y DeMartini, 1991). El cuadro clínico corresponde a una toxemia, generalmente de curso fatal (Ramírez et al., 1985; Moro, 1987), causando una severa enteritis hemorrágica o necrótica (Ameghino y DeMartini, 1991; Palacios, 2004).

Se tiene un conocimiento limitado sobre las toxinas de $C$. perfringens participantes en la patogenia de la enfermedad. Ramírez (1987) identificó cepas C. perfringens tipo A de casos típicos con capacidad de producir una enterotoxina (CPE), que lo condujo a proponer el posible rol patogénico de la CPE. Algunas de esas cepas, inoculadas en llamas, fueron capaces de inducir acumulaciones de fluido intestinal, responsabilizando a la CPE de los cambios observados. Esta propuesta ha sido contrastada por otros investigadores, quienes sugirieron principalmente la participación de las exotoxinas (Fowler, 1998; Prehn et al., 1999) y, tal vez, la presencia de una toxina secundaria, potencialmente patogénica, la beta2, detectada en algunas cepas aisladas de casos de enterotoxemia en alpacas (Bueschel et al., 2003; Ellis, 2006).

La tipificación del $C$. perfringens ha dependido, tradicionalmente, de inoculaciones de sobrenadantes bacterianos en animales de laboratorio; sin embargo, la disponibilidad de técnicas moleculares, como la reacción en cadena de la polimerasa (PCR) múltiple, que permite detectar de manera simultánea y en una misma reacción, la presencia de genes codificantes de las principales toxinas necesarias para arribar a una genotipificación [genes cpa ( toxina $\alpha$ ), cpb (toxina $\beta$ ), etx (toxina $\mathrm{g}$ ) e iap (toxina $\overline{3}$ ), incluyendo una subtipificación (gen cpe codificante de la enterotoxina y $c p b 2$ codificante de la toxina B2) (Songer y Meer, 1996; Yoo et al., 1997; Herholz et al., 1999; Baums et al., 2004). Esta técnica es una herramienta de diagnóstico rápida y confiable para determinar genotipos y subtipos de $C$. perfringens, en contraposición con la seroneutralización evidenciada en letalidad de ratones, técnica invasiva e incapaz de detectar bajos niveles de toxinas o cepas que no producen toxinas in vitro (Petit et al., 1999; Baums et al., 2004).

El presente estudio describe el aislamiento de ADN microbiano de 47 aislamientos de $C$. perfringens obtenidos de alpacas neonatas muertas por enterotoxemia y sometidos a amplificaciones moleculares con el objetivo de genotipificar y subtipificar usando la técnica de PCR múltiple. 


\section{Materiales y MéTodos}

\section{Muestras}

Se obtuvieron 47 muestras de intestino de alpacas neonatas con diagnóstico de enterotoxemia en el año 2005. Los animales provenían de cuatro hatos alpaqueros localizados sobre los $3500 \mathrm{msnm}$ en los departamentos de Cusco y Puno. El diagnóstico de la enfermedad se hizo de acuerdo a características epidemiológicas, signos clínicos, lesiones anatomopatológicas e histopatológicas. Las muestras consistieron en segmentos de intestino delgado afectado y ligado en sus extremos para ser transportadas a la Unidad de Biología Molecular y Genética (Facultad de Medicina Veterinaria, Universidad Nacional Mayor de San Marcos), Lima, en bolsas con tetraborato de sodio como preservante.

\section{Aislamiento e Identificación de $C$. perfringens}

Las muestras fueron cultivadas en caldo tioglicolato e incubadas a $45^{\circ} \mathrm{C}$ por $48 \mathrm{~h}$ en ambiente de anaerobiosis. Luego se tomaron alícuotas del medio para ser sembradas en agar sangre e incubadas bajo las mismas condiciones. Las colonias de $C$. perfringens fueron identificadas en base a características morfológicas de la colonia, patrón hemolítico, coloración Gram y reacción negativa a la catalasa. Estos aislados fueron luego sembrados en agar yema de huevo e incubadas a $37^{\circ} \mathrm{C}$ durante $18-24 \mathrm{~h}$ bajo anaerobiosis para evidenciar la reacción de Nagler. Finalmente, los aislados positivos fueron reactivados en caldo tioglicolato y conservados a $-20{ }^{\circ} \mathrm{C}$ para los análisis moleculares.

\section{Extracción de ADN Bacteriano}

Para la extracción de ADN bacteriano se utilizó $5 \mathrm{ml}$ de cultivo en caldo tioglicolato. Se les centrifugó a $13000 \mathrm{rpm}$ por $10 \mathrm{~min}, \mathrm{y}$ el precipitado fue re-suspendido en $800 \mu 1$ de EDTA $50 \mathrm{mM}$ más $200 \mu \mathrm{l}$ de lisozima. Se mezcló suavemente, se incubó a baño maría a $37^{\circ} \mathrm{C}$ durante $1-2 \mathrm{~h}$ y se centrifugó a 13000 rpm por $10 \mathrm{~min}$. El precipitado resultante fue utilizado para la extracción de ADN con el kit comercial "DNA Purification Kit" (Promega, Madison, USA), siguiendo las indicaciones del fabricante. El ADN extraído fue almacenado a $-20^{\circ} \mathrm{C}$.

\section{PCR Múltiple}

Para determinar los genotipos y subtipos de los aislados de C. perfringens, se utilizó la técnica de PCR múltiple descrita por Baums et al. (2004) con algunas modificaciones: la mezcla de PCR múltiple consistió en $15 \mu \mathrm{M}$ de los cebadores CPA5L y CPA5R, $9 \mu \mathrm{M}$ de los cebadores CPBL y CPBR, 5.3 $\mu \mathrm{M}$ de los cebadores CPEL y CPER, $7.5 \mu \mathrm{M}$ de los cebadores CPETXL y CPETXR, 7.5 $\mu \mathrm{M}$ de los cebadores CPIL y CPIR, $9 \mu \mathrm{M}$ de los cebadores CPB2L y CPB2L, $50 \mathrm{mM}$ de $\mathrm{KCl}, 10 \mathrm{mM}$ de Tris $\cdot \mathrm{Cl}$ (pH 8.3), $1.5 \mathrm{mM}$ de $\mathrm{MgCl}_{2}, 250 \mu \mathrm{M}$ de cada dNTP, 3.8 U de Taq polimerasa (Promega, Madison, USA) y 50 ng de ADN bacteriano. La amplificación se hizo en un termociclador 2720 (Applied Biosystems) a través de una desnaturalización inicial de 4 min a $95^{\circ} \mathrm{C}, 35$ ciclos de $1 \mathrm{~min}$ a $95{ }^{\circ} \mathrm{C}, 1 \min$ a $55^{\circ} \mathrm{C}$ y $1.5 \min$ a $72{ }^{\circ} \mathrm{C}$, y finalmente una etapa de extensión de $7 \mathrm{~min}$ a $72{ }^{\circ} \mathrm{C}$. Los productos de PCR se separaron mediante electroforesis en gel de agarosa al $2 \%$ en buffer TBE $0.5 \mathrm{X}$ y teñidos con bromuro de etidio. Las bandas amplificadas fueron visualizadas y fotografiadas bajo iluminación UV. Para la estandarización de la técnica se utilizaron como controles positivos cepas de la Colección Española de Cultivos Tipo (CECT): C. perfringens tipo A (CECT 563) y C. perfringens tipo C (CECT 820), mientras que $C$. septicum y $C$. chauvoei sirvieron como controles negativos.

\section{Genotipificación y Subtipificación}

La genotipificación de C. perfringens (tipos A, B, C, D, y E) se realizó en base a la presencia de los genes $c p a, c p b$, etx e iap, según la clasificación establecida por Petit et 
Cuadro 1. Determinación de genotipos y subtipos de cepas de referencia (Clostrium perfringens CETC 563 y CECT 820) y controles negativos (C. chauvoei y C. septicum) según la presencia (+) y ausencia (-) de gen es $c p a, c p b$, etx, iap, cpe y cpb2

\begin{tabular}{lcccccccc}
\hline Cepas de referencia & $c p a$ & $c p b$ & etx & iap & Genotipo & cpe & cpb2 & Subtipo \\
\hline $\begin{array}{l}\text { C. perfringens tipo A } \\
\text { (CECT 563) }\end{array}$ & + & - & - & - & $\mathrm{A}$ & - & - & cpe $^{-}$cpb2- \\
$\begin{array}{l}\text { C. perfringens tipo C } \\
\text { (CECT 820) }\end{array}$ & + & + & - & - & $\mathrm{C}$ & - & - & cpe $^{-}$cpb2- \\
$\begin{array}{l}\text { C. chauvoei } \\
\text { C. septicum }\end{array}$ & - & - & - & - & - & - & - & - \\
\hline
\end{tabular}

Cuadro 2. Frecuencias y porcentajes de los genotipos y subtipos de $C$. perfringens aislados de crías de alpacas muertas por enterotoxemia en el Perú (2005)

\begin{tabular}{|c|c|c|c|c|c|c|c|}
\hline Genotipo & & & A & & & $\mathrm{C}$ & \\
\hline Genes & & & cpa & & & $c p a, c p b$ & \\
\hline \multirow{2}{*}{ Aislados } & $\mathrm{N}^{\circ}$ & \multicolumn{3}{|c|}{46} & \multicolumn{3}{|c|}{1} \\
\hline & $\%$ & & 97.9 & & & 2.1 & \\
\hline \multirow{2}{*}{ Subtipo } & & $c p e^{-}$ & $c p e^{+}$ & cpe- & $c p e^{-}$ & $c p e^{+}$ & $c p e^{-}$ \\
\hline & & $c p b 2^{-}$ & $c p b 2^{-}$ & $c p b 2^{+}$ & $c p b 2^{-}$ & $c p b 2^{-}$ & $c p b 2^{+}$ \\
\hline \multirow{2}{*}{ Aislados } & N. ${ }^{\circ}$ & 33 & -- & 13 & -- & 1 & -- \\
\hline & $\%$ & 71.7 & -- & 28.3 & -- & 100.0 & -- \\
\hline
\end{tabular}

al. (1999). La subtipificación se realizó detectando la presencia de los genes сре у сpb2, según lo descrito por Garmory et al. (2000).

\section{Resultados}

Se logró aislar C. perfringens en las 47 muestras intestinales de crías muertas por enterotoxemia. Todos los aislados fueron positivos a la tinción Gram, presentaron una doble hemólisis, fueron catalasa negativa y, mayoritariamente (42/47), fueron positivas a la Reacción de Nagler.
Los resultados obtenidos por la PCR múltiple de las cepas de referencia de $C$. perfringens tipo A y $\mathrm{C}$ amplificaron genes correspondieron a los tipos $\mathrm{A}$ y $\mathrm{C}$, respectivamente, mientras que las cepas de $C$. chauvoei y C. septicum no evidenciaron amplicones similares (Cuadro 1). La amplificación genética determinó que el 97.9\% (46/47) de los aislamientos de $C$. perfringens fueron del genotipo A y el restante $(2.1 \%)$ correspondió al genotipo C. El 71.7\% (33/46) de los aislamientos de $C$. perfringens con genotipo A que no tenían los genes secundarios cpe y $\beta 2$ correspon- 
dieron al subtipo $c p e^{-v o} c p b 2^{-v o}$ y el 28.3\% (13/ 46) restante correspondió al subtipo cpe${ }^{v o} \mathrm{Cpb2}^{+v o}$ (presencia del gen $c p b 2$ ). El único C. perfringens genotipo $\mathrm{C}$ aislado presentó el gen de la enterotoxina (subtipo $c p e^{+v o} c p b 2-$ ${ }^{v o}$ ) (Cuadro 2).

\section{Discusión}

La técnica de PCR múltiple permitió la geno y subtipificación de los 47 aislados de C. perfringens obtenidos de casos confirmados de enterotoxemia en neonatos de alpacas, evidenciando ser una herramienta de diagnóstico rápida y confiable (Petit et al., 1999; Baums et al., 2004), contrastando con el método de tipificación clásico de la prueba de seroneutralización in vivo, basada en la letalidad de ratones o por dermato-necrosis en cobayos. Estas ventajas han permitido a la PCR múltiple ser empleada en forma exitosa en este estudio y por otros investigadores interesados en elucidar la participación de las toxinas de $C$. perfringens en procesos entéricos en lechones (Kanakaraj et al., 1998; Garmory et al., 2000; Waters et al., 2003), enteritis necrótica en pollos (Engstrom et al., 2003; Gholamiandekhordi et al., 2005), enterotoxemia en corderos (Gkiourtzidis et al., 2001; Kalender et al., 2005), enterotoxemia en ciervos (Embury-Hyatt et al., 2005), tiflocolitis en caballos (Herholz et al., 1999) y en intoxicación de origen alimentaria en humanos y diarreas asociadas a antibióticos en humanos (Sparks et al., 2001).

E1 97.9\% de los aislamientos correspondieron al genotipo A, concordando con otros resultados (Moro, 1987; Ramírez, 1987), quienes mediante la seroneutralización determinaron al tipo A del C. perfringens como el principal agente causal de la enterotoxemia en alpacas. Los aislados pertenecientes al genotipo A y de acuerdo a la presencia de genes secundarios fueron agrupados en subtipos, siguiendo la clasificación propuesta por Garmory et al. (2000), determinando que el $71.7 \%$ fueron del subtipo $c p e^{- \text {vo }} c p b 2^{- \text {-vo }}$, es decir, cepas incapaces de producir enterotoxina o toxina $\beta 2$, mientras que los 13 restantes correspondieron al subtipo cpe $^{-}$ ${ }^{\mathrm{vo}} \mathrm{Cpb2} 2^{+\mathrm{vo}}$, con sólo la capacidad de producir la toxina $\beta 2$.

En ninguno de los $46 / 47$ aislados se logró amplificar el gen cpe productor de enterotoxina, sugiriendo que la enterotoxina de C. perfringens no participaría en el desarrollo de la enterotoxemia de las alpacas. Estos resultados son similares a otros estudios donde no se detectó la presencia del gen cpe en aislamientos de $C$. perfringens provenientes de alpacas con problemas entéricos (Garmory et al., 2000; Bueschel et al., 2003; Baums et al., 2004; Ellis, 2006), e incluso asociados a casos de muertes súbitas (Sawires y Songer, 2006). La ausencia del gen cpe también ha sido demostrado en reportes de casos de enterotoxemia de terneros, cabras, venados y corderos (Manteca et al., 2002; Dray, 2004; Embury-Hyatt et al., 2005; Kalender et al., 2005), así como en cuadros descritos como disentería hemorrágica en corderos (Gkiourtzidis et al., 2001), enteritis necrótica en pollos (Engstrom et al., 2003; Gholamiandekhordi et al., 2005), enteritis necrótica en lechones (Kanakaraj et al., 1998; Garmory et al., 2000, Waters et al., 2003) y tiflocolítis en caballos (Herholz et al., 1999). La ausencia del gen cpe llama enormemente la atención, pues la enterotoxina fue siempre considerada como el principal factor de virulencia del C. perfringens en la patogénesis de la enterotoxemia en las alpacas (Ramírez, 1987), pese a que no se descartaron en dichos estudios la presencia de otras toxinas (toxinas y $\beta 2$ ).

Por otro lado, el efecto patogénico de la enterotoxina no encaja con los cuadros de enterotoxemia descritos en alpacas, pues la producción de esta toxina está frecuentemente asociada a problemas de intoxicación alimentaria que ocasionan diarreas profusas, esporádicas y autolimitantes en individuos inmunocompetentes (McClane, 2000; Sparks et al., 2001). Los casos clásicos de enterotoxemia en alpacas raramente se observan o se asocian con diarreas y cuando 
ocurren son generalmente a consecuencia de infecciones mixtas por E. coli (Ameghino y DeMartini, 1991).

La alta frecuencia de $C$. perfringens genotipo A subtipo $c p e^{-v o}$ y $c p b 2^{-v o}(71.7 \%)$ tiende a señalar que solamente la presencia del gen cpa y su expresión (toxina $\alpha$ ) sería suficiente para la producción de la enfermedad. Similares resultados fueron encontrados en estudios de geno y subtificación de $C$. perfringens aislados de casos de enteritis necrótica en pollos y en cerdos (Kanakaraj et al., 1998; Engstrom et al., 2003; Gholamiandekhordi et al., 2005; Kalender y Ertas, 2005), enterotoxemia en corderos y terneros (Songer, 1996; Dennison et al., 2005; Kalender et al., 2005) y gastroenteritis hemorrágica en perros (Songer, 1996).

Este trabajo reporta por primera vez en el Perú la presencia de $C$. perfringens genotipo A subtipo $\beta 2$-toxigénico aislados de casos de enterotoxemia de alpacas, evidenciando la posible participación de esta toxina en la etiopatogénesis de la enfermedad. Similares hallazgos han sido reportados por otros investigadores, quienes detectaron la presencia del gen $c p b 2$ en $18.6 \%(8 / 43)$ de los C. perfringens aislados de alpacas con problemas entéricos (Bueschel et al., 2003).

La presencia del gen $c p b 2$ ha sido asociada con problemas entéricos en varias especies; por ejemplo, cerca del $90 \%$ de $C$. perfringens $\beta 2$ aislados en lechones se encuentran asociados con enteritis necrótica, mientras que en aislamientos de cerdos clínicamente sanos el gen $c p b 2$ no está presente o no ha sido detectado (Garmory et al., 2000; Bueschel et al., 2003). Similarmente, se reporta que el 50 a $75 \%$ de los aislamientos de C. perfringens $\beta 2$ de casos de tiflocolitis en potros (Herholz et al., 1999; Garmory et al., 2000), así como en cuadros de enterotoxemia en terneros, cabras y venados (Manteca et al., 2002; Dray, 2004; Embury-Hyatt et al., 2005) y disentería hemorrágica en corderos (Gkiourtzidis et al., 2001) demuestran una posible asociación patológica con cepas $c p b 2$ positivas.
En el presente estudio, se aisló solamente una cepa $(2.1 \%)$ perteneciente al genotipo $\mathrm{C}$, corroborando la eventualidad de su presencia en casos patológicos (Moro, 1987). Curiosamente, este genotipo fue también el único subtipo enterotoxigénico $\left(c p e^{+v o} c p b 2^{-v o}\right)$. No se lograron aislar los genotipos B ni D como los descritos en Chile (Prehn et al., 1999) y en EEUU (Fowler, 1998), respectivamente.

\section{ConClusiones}

- $\quad$ El C. perfringens genotipo A y subtipo $c p e^{-v o} c p b 2^{-v o}$ (no enterotoxigénico ni $\beta 2$ toxigénico) es el genotipo y subtipo más prevalente en casos de enterotoxemia en alpacas.

- Se reporta por primera vez en el Perú, la presencia del gen $c p b 2$ y la casi ausencia del gen codificante de la enterotoxina.

- Los resultados apuntan básicamente a la toxina a y, posiblemente, a la toxina b2 como los principales factores de virulencia asociados en la enterotoxemia de las alpacas.

\section{Agradecimientos}

Los autores agradecen a los médicos veterinarios y criadores que participaron en la toma de muestras en los hatos alpaqueros localizados en la zona sur del Perú. El estudio fue financiado por el Consejo Nacional de Ciencia y Tecnología e Innovación (CONCYTEC) (Desarrollo de vacunas de nueva generación para prevenir enterotoxemia en alpacas), el Consejo Superior de Investigaciones de la Universidad Nacional Mayor de San Marcos (CSIUNMSM), INCAGRO (Identificación y selección de marcadores moleculares de resistencia genética a enterotoxemia y neumonía en crías de alpacas) y CONOPA - Instituto de Investigación y Desarrollo de Camélidos Sudamericanos. 


\section{LiTERATURA CitadA}

1. Ameghino E, DeMartini J. 1991. Mortalidad en crías de alpacas. Bol Divul IVITA, Perú: $128 \mathrm{p}$.

2. Baums CG Schotte U, Amtsberg G, Goethe R. 2004. Diagnostic multiplex PCR for toxin genotyping of Clostridium perfringens isolates. Vet Microbiol 100: 11-16.

3. Bueschel DM, Jost BH, Billington SJ, Trinh HT, Songer JG. 2003. Prevalence of $c p b 2$, encoding beta 2 toxin, in Clostridium perfringens field isolates: correlation of genotype with phenotype. Vet Microbiol 94: 121-129.

4. Carbajal M. 1974. Determinación de las causas de mortalidad en crías de alpaca en el departamento de Puno en las campañas ganaderas de 1972 y 1973. Tesis de Médico Veterinario Zootecnista. Puno: Facultad de Medicina Veterinaria y Zootecnia, Univ Nacional del Altiplano. $44 \mathrm{p}$.

5. Dennison AC, Van Metre DC, Morley PS, Callan RJ, Plamplin EC, Ellis RP. 2005. Comparison of the odds of isolation, genotypes, and in vivo production of major toxins by Clostridium perfringens obtained from the gastrointestinal tract of dairy cows with hemorrhagic bowel syndrome or left-displaced abomasum. JAVMA 227: 132-138.

6. Dray T. 2004. Clostridium perfringens type $A$ and $\beta 2$ toxin associated with enterotoxemia in a 5-week-old goat. Can Vet J 45: 251-253.

7. Ellis RP. 2006. Clostridium perfringens enteritis. In: The complete alpaca book. $2^{\text {nd }}$ ed. Hoffman E (ed). California: Bonny Doun Press. p 464467.

8. Embury-Hyatt CK, Wobeser G, Simko E, Woodbury MR. 2005. Investigation of a syndrome of sudden death, splenomegaly, and small intestinal hemorrhage in farmed deer. Can Vet $\mathrm{J}$ 46: 702-708.
9. Engström BE, Fermér C, Lindberg A, Saarinen E, Baverud V, Gunnarsson A. 2003. Molecular typing of isolates of Clostridium perfringens from healthy and diseased poultry. Vet Microbiol 94: 225-235.

10. Fowler ME. 1998. Medicine and surgery of South American camelids. $2^{\text {nd }}$ ed. Iowa: Iowa State University Press. $549 \mathrm{p}$.

11. Garmory HS, Chanter N, French NP, Bueschel D, Songer JG, Titball RW. 2000. Ocurrence of Clostridium perfringens $\beta 2$-toxin amongst animals, determinated using genotyping and subtyping PCR assays. Epidemiol Infect 124: 61-67.

12. Gholamiandekhordi AR, Ducatelle R, Heyndrickx M, Haesebrouck F, Immerseel FV. 2005. Molecular and phenotypical characterization of Clostridium perfringens isolates from poultry flocks with different disease status. Vet Microbiol 113: 143-152.

13. Gkiourtzidis K, Frey J, BourtziHatzopoulou E, Iliadis N, Sarris K. 2001. PCR detection and prevalence of $\alpha-, \beta-, \beta 2-, 9-, \overline{3}-$ and enterotoxin genes in Clostridium perfringens isolated from lambs with clostridial dysentery. Vet Microbiol 82: 39-43.

14. Herholz C, Miserez R, Nicolet J, Frey J, Popoff M, Gubert M, Gerber $H$, Straub R. 1999. Prevalence of $\beta 2$ toxigenic Clostridium perfringens in horses with intestinal disorders. J Clin Microbiol 37: 358-361.

15. Kalender H, Ertas HB, Ceninkaya B, Muz A, Aíslan N, Kilic A. 2005. Typing of isolates of Clostridium perfringens from healthy and diseased sheep by multiplex PCR. Vet Med-Czech 50: 439442.

16. Kalender H, Ertas HB. 2005. Isolation of Clostridium perfringens from chickens and detection of the alpha toxin gene by polymerase chain reaction (PCR). Turk J Vet Anim Sci 29: 847-851. 
17. Kanakaraj R, Harris DL, Songer JG, Bosworth B. 1998. Multiplex PCR assay for detection of Clostridium perfringens in feces and intestinal contents of pigs and swine feed. Vet Microbiol 63:29-38.

18. Manteca C, Daube G, Jauniaux T, Linden A, Pirson V, Detilleux J, Ginter $A$, et al. 2002. A role for the Clostridium perfringens $\beta 2$ toxin in bovine enterotoxaemia? Vet Microbiol 86: 191-202.

19. McClane BA. 2000. Clostridium perfringens enterotoxin and intestinal tight junctions. Trends Microbiol 8: 145146.

20. Moro M. 1966. Enfermedades infecciosas de las alpacas. Enterotoxemia, diarrea bacilar producida por el Clostridium welchii tipo A. Rev Fac Med Vet, UNMSM.18-20: 85-87.

21. Palacios C. 2004. Caracterización anatomopatológica de enteropatías causantes de mortalidad en crías de alpaca. Tesis de Médico Veterinario. Lima: Facultad de Medicina Veterinaria, Univ Nacional Mayor de San Marcos. 164 p.

22. Petit L, Gibert M, Popoff M. 1999. Clostridium perfringens: toxinotype and genotype. Trends Microbiol 7: 104110.

23. Prehn N, Saez S, Arraigada M. 1999. Estudios microbiológicos y clínicos de enterotoxemia por Clostridium perfringens en camélidos sudamericanos. II Cong. Mundial sobre Camélidos. Cusco, Perú. p 140.

24. Ramírez A, Huamán D, Ellis RP. 1985. Enterotoxemia de la alpaca. Programa colaborativo de apoyo a la investigación en rumiantes menores. INIPA y SR-CRSP. Reporte Técnico N. ${ }^{\circ}$ 63. Lima. 17 p.

25. Ramírez A. 1987. Alpaca Clostridium perfringens type A enterotoxemia: Purification and assays of the enterotoxin. PhD Thesis. USA: Colorado State University. 201 p.

26. Sawires SY, Songer JG. 2006. Clostridium perfringens: Insight into virulence evolution and population structure. Anaerobe 12: 23-43.

27. Songer JG, Meer RR. 1996. Genotyping of Clostridium perfringens by polymerase chain reaction is a useful adjunct to diagnosis of clostridial enteric disease in animals. Anaerobe 2: 197-203.

28. Songer JG. 1996. Clostridial enteric diseases of domestic animals. Clin Microbiol Rev 9: 216-234.

29. Sparks SG Carman RJ, Sarker MR, McClane BA. 2001. Genotyping of enterotoxigenic Clostridium perfringens fecal isolates associated with antibiotic-associated diarrhea and food poisoning in North America. J Clin Microbiol 39: 883-888.

30. Waters M, Savoie A, Garmory HS, Bueschel D, Popoff MR, Songer JG, Titball RW, et al. 2003. Genotyping and phenotyping of beta2-toxigenic Clostridium perfringens fecal isolates associated with gastrointestinal disease in piglets. J Clin Microbiol 41: 3584-3591.

31. Yoo HS, Lee SU, Park KY, Park YH. 1997. Molecular typing and epidemiological survey of prevalence of Clostridium perfringens types by multiplex PCR. J Clin Microbiol 35: 228 232. 